\title{
The Role of Collagen Membrane as a Scaffold of Etchant for Regional Acceleratory Phenomenon
}

\author{
Seung-Woo Shin ${ }^{1}$, Sung-Woon Pyo ${ }^{2}$, Sun-Sook Bae ${ }^{1}$, Pil-Woo Lee ${ }^{1}$, \\ Hyun-A Heo ${ }^{2}$, Won Lee ${ }^{1}$
}

\begin{abstract}
1. Department of Oral and Maxillofacial Surgery, Uijeongbu St. Mary's Hospital, The Catholic Univeristy of Korea, Uijeongbu, Korea

2. Department of Oral and Maxillofacial Surgery, Bucheon St. Mary's Hospital, The Catholic University of Korea, Bucheon, Korea
\end{abstract}

\section{Corresponding Author}

\section{Won Lee, DDS, PhD}

Division of Oral and Maxillofacial Surgery, Department of Dentistry, Uijeongbu St. Mary's Hospital, 65-1 Geumoh-dong, Uijeongbu 480-717, Korea

TEL : +82-31-840-3184 FAX :+82-31-840-3185 E-mail: cmfs21@yahoo.com

Received for publication May 30, 2011; Returned after revision November 17, 2011;

Accepted for publication November 24, 2011

\section{- Abstract}

Purpose: The goal of this research is to find the role of collagen membrane, which can reduce physical damage, as a scaffold for possible alternative to the corticotomy which causes Regional Acceleratory Phenomenon (RAP).

Materials and Methods: The experiments were carried out on 12 New Zealand white rabbits, approximately $3.5 \mathrm{~kg}$ in bodyweight. We made an incision on the skin of the mandibular border and applied $37 \%$ phosphoric acid and collagen membrane to the mandibular bone surface of the first group (experimental group), and only phosphoric acid to the second group (control group). After 3 days, 1 week, and 2 weeks, 4 rabbits each were sacrificed and specimens were obtained. Each specimen was stained by H\&E and Tartrate-resistant acid phosphatase (TRAP), and histological changes were observed by light microscope.

Results: The demineralization of the experimental group was weak compared to the control group. It also showed a gradual increase of demineralization (after 3 days, 1 week, and 2 weeks) and the control group showed more extensive demineralization than the experimental group.

Conclusion: This study demonstrates the amount of demineralization as a result of using phosphoric acid, and as time went by, demineralization increased. The absorbable collagen membrane was used as a scaffold to increase bone demineralization effect and prevent dispersion to adjacent tissues, but rather the amount of bone demineralization decreased. Therefore, the role of collagen membrane as a scaffold for RAP was weak.

\section{- Key word : Corticotomy, Regional acceleratory phenomenon, Demineralization, Collagen membrane}

- J Kor Dent Sci. 2011; 4(2) : 39 - 45

(c) This is an open access article distributed under the terms of the Creative Commons Attribution Non-Commercial License (http:// creativecommons.org/licenses/by-nc/3.0) which permits unrestricted non-commercial use, distribution, and reproduction in any medium, provided the original work is properly cited. 


\section{Introduction}

As the intention to oral hygiene and aesthetics grows, the demand for adult orthodontic treatment has increased and universalized. One of the main disadvantages of conducting adult orthodontic treatment is that the treatment requires duration of 1 2 years or longer. Therefore, to shorten treatment duration and move teeth on an effective basis for adult orthodontic treatment, medication ${ }^{1,2)}$, magnetic field ${ }^{3)}$, resonance vibration ${ }^{4)}$, low-energy laser ${ }^{5,6)}$, and so forth have been studied. However, medication can affect the whole body with complications; if using magnetic field and vibration, the size of oral appliances is limited; for laser treat ${ }^{-}$ ment, there is little study on intensity and wavelength of laser output to facilitate tooth movement ${ }^{6}$.

Since tooth movement undergone along with surgical procedure shortens treatment duration from the usual orthodontic treatment, studies on such methods have been conducted. Kole ${ }^{7}$ stressed that, as cortical bone acted as the main resistance to tooth movement, the speed of tooth movement increased when blocking the continuity of cortical bone; and reported that when drilling a hole on the labial and lingual cortical bones around the dental root and imposing orthodontic force, tooth movement could be accelerated and the treatment duration can be shortened. Wilcko et al. ${ }^{8)}$ showed that when removing limited buccal and lingual cortical bones using a circular bar, the tooth movement was accelerated. Accelerated tooth movement and increased bone formation from demineralized bone in corticotomy is said to be due to Regional Acceleratory Phenomenon (RAP $)^{9,10)}$. RAP occurs within a few days after surgery; peaks in a month or two; and disappear within 6 to 24 months ${ }^{9,10)}$. The initial RAP shows increases in porosity of cortical bone and metabolism rate on trabecular bone surface due to the increased osteoclasts activities ${ }^{11)}$.

Likewise, rapid orthodontic treatment accompanied by corticotomy not only accelerates tooth movement, shortening the duration of treatment, but also reduces side effects such as protrusion of the root of tooth out of the alveolar bone when the moving tooth is accompanied by bone atrophy; or root resorption by excessive force. Such procedures, however, require surgeries, and physically damage teeth and adjacent periodontal tissue, possibly causing complications such as necrosis and resorption of the alveolar bone, gingival recession, teeth root and nerve damage, etc ${ }^{12}$.

If it is possible to demineralize the surface of the alveolar bone to induce RAP by the stimulation similar to corticotomy, the complications caused by mechanical bone removal can be reduced and a simple surgical treatment can facilitate tooth movement. As such, if it is possible to obtain effects similar to those of corticotomy by demineralizing cortical bone using acid, the procedure can be simplified and the physical damage to teeth and periodontal tissue can be reduced. The study by $\mathrm{Kim}^{13)}$ reported that even though acid demineralization could not bring about as much effect as mechanical removal in corticotomy, RAP phenomenon could be confirmed at the demineralized region, where cortical bone resorption, formation of new bone and osteoclasts activity occurred. With acid-demineralization of bone, the depth of demineralization increases according to acid etching time and acid concentration; however, compared with surgical bone removal, such demineralized amount is far smaller so sufficient demineralization time and scope expansion are necessary to induce RAP.

Therefore, to prevent acid harm to adjacent tissues and to increase demineralization effect, the study is to observe bone tissue changes using absorbable collagen membrane. Absorbable collagen membrane has effects of hemostasis, histocompatibility and wound healing, and is useful as a temporary barrier membrane for maxillary antral puncture or partially exposed regions.

This experiment is to confirm the usefulness of absorbable collagen membrane as a scaffold and the difference of bone demineralization when increasing acid applying time through animal tests to allow acid to remain on cortical bone for a sufficient length of time to elicit demineralization.

\section{Materials and Methods}

\section{Material}

\section{1) Experimental Animals}

The experiments were carried out on 12 female New Zealand white rabbits, approximately $3 \sim 4 \mathrm{~kg}$ in body weight, which were raised using solid animal feed and water under lighting and at a temperature adjusted to room temperature. 12 rabbits were classified into 4 each in 3 groups according to demineralization time $(3,7,14$ days). The e group received phosphoric acid and absorbable collagen membrane (Collatape, Zimmer Dental, Carlsbad, CA, USA) on the right mandibula and the control group received only phosphoric acid on the left mandibula. The animal experi- 
ments were conducted with the approval obtained from Uijeongbu St. Mary's Hospital Animal Care and Use Committee (UJA 2009-16A).

\section{2) Experimental Materials}

Acid used for experimental animals applied $0.1 \mathrm{cc}$ of $37 \%$ phosphoric acid (Etch-37, pH 0.1, Bisco, Chicago, IL, USA), the same as in the research method by $\mathrm{Kim}^{13)}$, and absorbable collagen membrane was used as a scaffold. Post-operatively, absorbable sutures (Vicryl 4-0, Ethicon, Kirkton, Scotland, UK) were used.

\section{Procedure}

\section{1) Operation Method}

For anesthesia, an intramuscular injection of Zolazepam 15 $\mathrm{mg} / \mathrm{kg}$, Tiletamina $15 \mathrm{mg} / \mathrm{kg}$ (Zoletil, Virbac Laboratories, Carros, France) and Xylazine $5 \mathrm{mg} / \mathrm{kg}$ (Xylazine 20, Kepro BV, Deventer, Netherlands) induced general anesthesia. After disinfecting the submandibular border of rabbits with betadine solution, $40 \mathrm{~mm}$ of skin, from mandibular angle to premolar along the submandible, was incised and dissected subcutaneous tissue and periosteum in full-thickness flap exposing the mandible. On the bone surface of left side of the root of mandibular $1^{\text {st }}$ molar, $0.1 \mathrm{cc}$ of $37 \%$ phosphoric acid was applied; on the right side, $0.1 \mathrm{cc}$ of $37 \%$ phosphoric acid (Bisco) was applied to $1.0 \times 0.5 \mathrm{~cm}$ collagen membrane and then the flaps were sutured using 4-0 Vicryl. To prevent post-operative infection, $4 \mathrm{mg} / \mathrm{kg}$ Gentamicin (Kukje Pharm Inc., Cheonan, Korea) and $1 \mathrm{mg} / \mathrm{kg}$ Ketoprofen (Bukwang Pharm, Seoul, Korea) were intramuscularly injected once a day for 3 days (Figs. 1, 2).

After the experiment duration, rabbits were put in general anesthesia and sacrificed through euthanasia by injecting excessive intravenous injection of KCI (Huons, Seoul,

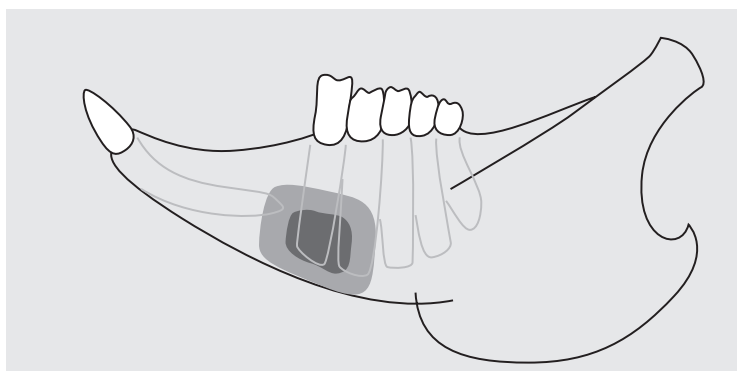

Figure 1. Experimental design. Exposed cortical bone area (gray area, control site) and acid etching area (black area, experimental site).

Seung-Woo Shin, et al: The Role of Collagen Membrane as a Scaffold of Etchant for Regional Acceleratory Phenomenon. J Kor Dent Sci 2011.
Korea).

2) Tissue Specimen Preparation and Observation

Experimental animals were sacrificed at 3 days, 1 week and 2 weeks post-op; the mandibula was exposed in the same fashion as the initial surgery; with the incision including adjacent bone; and fixed in paraformaldehyde. Fixed specimens were soaked in 10\% EDTA at room temperature for about 3 weeks; and demineralized on Shaker (Compact Rocker, FINE PCR Co., Gunpo, Korea). The specimens were cut horizontally to the occlusal plane at mandibular angle. $4 \mu \mathrm{m}$ thick cut sections were stained with H\&E and observed with a light microscope (IX71 Inverted Microscope, Olympus, Tokyo, Japan).

\section{3) Measurement of Demineralized Area}

First, section views of tissue slides were taken by digital camera (DP70, Olympus); the area of demineralized cortical bone was measured using Paint net (dot PDN Inc, Seattle, WA, USA). By comparing and compensating such measurements with actual measurements at 100 times magnification, the area equivalent to one pixel was measured and then the total area was calculated by multiplying the total number of pixels on the demineralized region. Statistical differences between the demineralized area of control and experimental site were assessed by paired t-test (Excel 2010, Microsoft Co., Redmond, WA, USA). Statistical significance was set at $\mathrm{P} \leq 0.05$.

\section{4) Observation of Tartrate-Resistant Acid Phosphatase} (TRAP) Positive Cells

TRAP staining to identify osteoclasts was performed using previously published methods ${ }^{14)}$. Briefly describing the process, cut sections were processed in the mixture of 24 $\mathrm{mg}$ red violet salt (Sigma, St. Louis, MO, USA) and $30 \mathrm{ml}$ of $0.1 \mathrm{~mol} / \mathrm{l}$ acetate buffer ( $\mathrm{pH} 5.2$ ) including $4 \mathrm{mg}$ naphthol
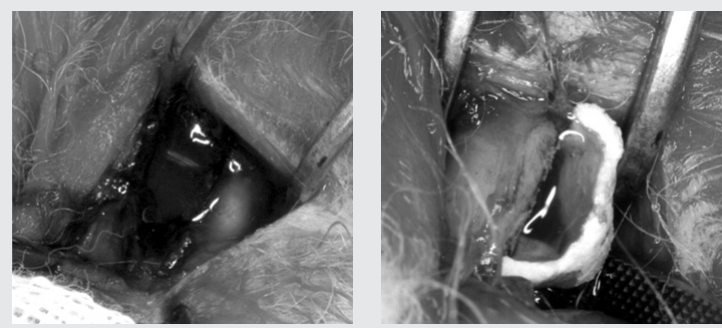

Figure 2. Phosphoric acid was directly applied on the bone surface of the mandibular angle (control) and covered with collagen membrane (experimental side).

Seung-Woo Shin, et al: The Role of Collagen Membrane as a Scaffold of Etchant for Regional Acceleratory Phenomenon. J Kor Dent Sci 2011. 
AS-BI phosphate $4 \mathrm{mg}$ (Sigma) and $0.3 \mathrm{mmol} / \mathrm{l}$ tartrate $(\mathrm{pH}$ 5.0) (Sigma) at $37^{\circ} \mathrm{C}$ for 15 minutes. Cut sections were stained with hematoxylin for comparison. TRAP positive cells were observed with a light microscope.

\section{Results}

\section{Histological Observations}

As similar to the histological observations shown in corticotomy, when demineralizing cortical bone with acid, the continuity of trabeculae was lost during the initial demineralization period, and fibrous tissue and new bone deposition into the lost region were observed. From the normal bone tissue, only lamellar bone was observed; from demineralized tissue, immature bone was observed on the cortical bone surface. The longer acid was applied, the broader of demineralized cortical bone was; and infiltration of fibrous tissue and inflammatory cells into this broadened area increased.

Comparing demineralized areas as time went by, the 7-day group showed demineralization areas broader than those of the 3-day group's; and the 14-day group showed much broader demineralization areas (Fig. 3).

\section{Analysis of Demineralized Area}

To comparatively analyze demineralization degree between

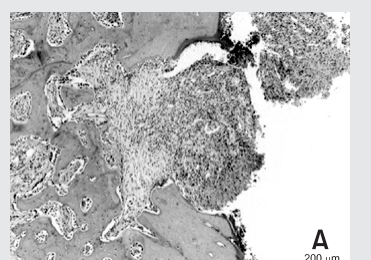

$\underset{200 \mu \mathrm{m}}{\mathbf{A}}$

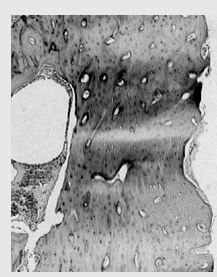

$\underset{200 \mu \mathrm{mm}}{\mathbf{B}}$
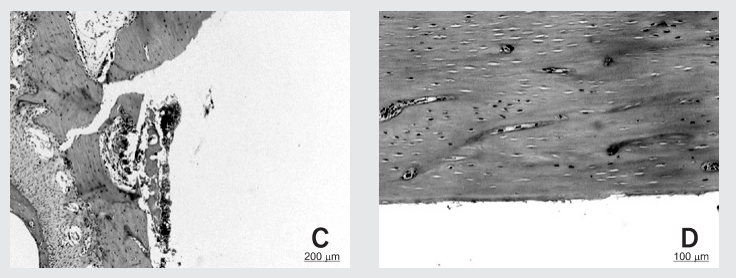

Figure 3. These photographs show the shape of demineralization as a result of the use of phosphoric acid, and the increase of demineralization progressively increasing, after 3 days $(A), 7$ days $(B), 14$ days $(C)$ intact cortical bone on the nonsurgical side (D). H\&E stain (x200).

Seung-Woo Shin, et al: The Role of Collagen Membrane as a Scaffold of Etchant for Regional Acceleratory Phenomenon. J Kor Dent Sci 2011. two groups, the area of demineralized cortical bone region was measured. As seen in Table 1 and Fig. 4, the demineralization amount increased as time went by. While there was not that much difference between the Experimental group and control group in groups 1 and 2, the control group in group 3 showed a slightly larger demineralization area than the experimental group in group 3; although it was not statistically significant $(\mathrm{P}>0.05)$ (Fig. 5).

\section{Observation of TRAP Positive Cells}

In the experiment conducted to determine the activity of osteoclasts, osteoclasts were observed in the demineralized region in both the experimental and control groups. Moreover, the longer the acid was applied, the fewer osteoclasts were observed; for the same period, the difference between the experimental group and control group was not significant. The demineralized region was replaced by woven bone and healed, and osteoclasts were observed around the woven bone (Fig. 6).

\begin{tabular}{|c|c|c|c|c|c|c|c|}
\hline & & 1 & 2 & 3 & 4 & $\begin{array}{c}\text { Means } \pm S D \\
\left(\mathrm{~mm}^{2}\right)\end{array}$ & t-test \\
\hline \multirow{2}{*}{$\begin{array}{c}\text { Group } 1 \\
\text { (3 days) }\end{array}$} & $\mathrm{C}$ & 0.14 & 0.05 & 0.05 & 0.02 & $0.07 \pm 0.05$ & \multirow[t]{2}{*}{0.62} \\
\hline & $E$ & 0.03 & 0.05 & 0.06 & 0.05 & $0.05 \pm 0.01$ & \\
\hline \multirow{2}{*}{$\begin{array}{l}\text { Group } 2 \\
\text { (7 days) }\end{array}$} & C & 0.08 & 0.09 & 0.07 & 0.18 & $0.11 \pm 0.04$ & \multirow[t]{2}{*}{0.40} \\
\hline & E & 0.20 & 0.07 & 0.14 & 0.15 & $0.14 \pm 0.05$ & \\
\hline \multirow{2}{*}{$\begin{array}{l}\text { Group } 3 \\
\text { (14 days) } \\
\end{array}$} & C & 0.56 & 0.19 & 0.36 & 0.26 & $0.34 \pm 0.14$ & \multirow[t]{2}{*}{0.21} \\
\hline & E & 0.26 & 0.20 & 0.20 & 0.25 & $0.23 \pm 0.03$ & \\
\hline \multicolumn{8}{|c|}{ C: control group, E: experiment group. } \\
\hline
\end{tabular}

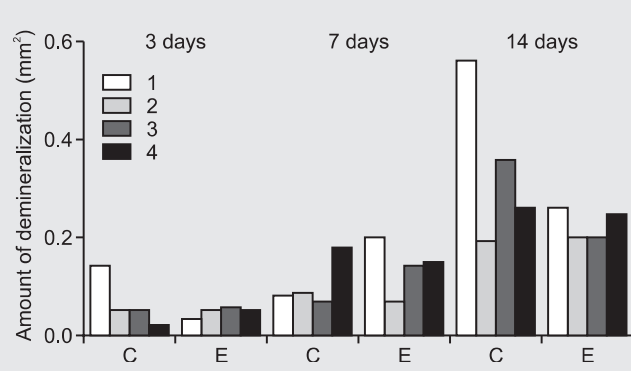

Figure 4. Comparison of the amount of area of demineralization according to the passage of time between the experimental and control groups, C (control group), E (experiment group).

Seung-Woo Shin, et al: The Role of Collagen Membrane as a Scaffold of Etchant for Regional Acceleratory Phenomenon. J Kor Dent Sci 2011. 


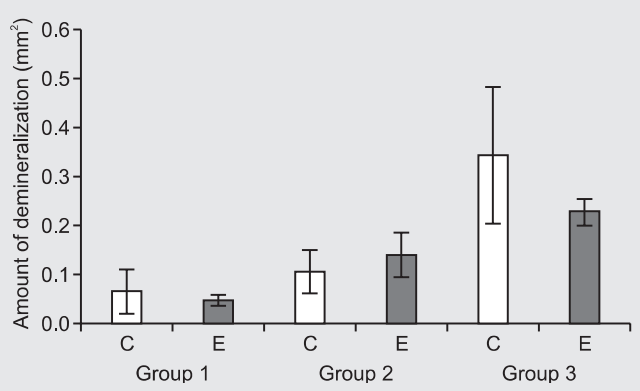

Figure 5. Comparison of the average amount of area of demineralization according to the passage of time between the experimental and control groups, C (control group), E (experiment group).

Seung-Woo Shin, et al: The Role of Collagen Membrane as a Scaffold of Etchant for Regional Acceleratory Phenomenon. J Kor Dent Sci 2011.

\section{Discussion}

In this experiment, to replace corticotomy, phosphoric acid was applied to mandibular to obtain effects similar to corticotomy through demineralization by acid, the amount of demineralization was compared over time after acid application, and a method to increase the amount of demineralization efficiently was sought.

The experiment adopted the method of demineralization using acid to replace corticotomy, and phosphoric acid was selected from available dental treatment acids that include phosphoric acid, hydrofluoric acid, maleic acid and EDTA. EDTA is difficult to handle as there is no gel-type EDTA and only liquid-type EDTA is used for endodontic treatment; in addition, since the degree of demineralization is weak (when applied to teeth) compared to phosphoric acid, EDTA is inappropriate material for demineralization of cortical bone ${ }^{15)}$. Maleic acid is rarely on the market; and has weak bonding strength when applied to teeth compared to phosphoric acid. No case of hydrofluoric acid application to human body has been reported. To that end, the experiment used phosphoric acid, which was deemed most appropriate. Evaluating the demineralization degree of teeth using phosphoric acid, $27.1 \mu \mathrm{m}$ was demineralized when applying $37 \%$ phosphoric acid to enamel for 60 seconds $^{16)} ; 7.4 \mu \mathrm{m}$ was demineralized when applying $35 \%$ phosphoric acid to dentin for 60 seconds; $13.1 \mu \mathrm{m}$ when applying the acid while stirring with an instrument ${ }^{17}$. Comparing components of teeth and bone, $97 \%$ of enamel and $69 \%$ of dentin consist of minerals. As teeth can be demineralized by acid, bone is also supposed to be demineralized because $50 \%$ of bone

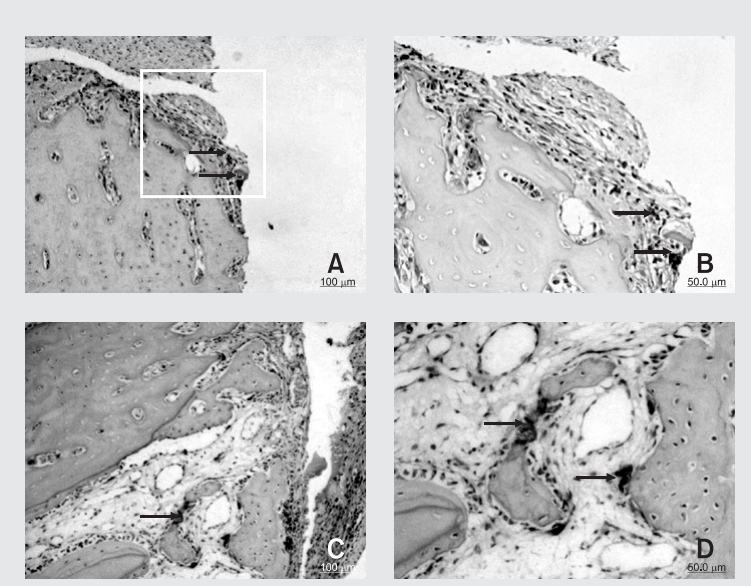

Figure 6. Activity of osteoclast was observed. The arrows indicate the TRAP positive cells. Left (A, C) TRAP stain (x200), Right $(B, D)$ TRAP stain $(x 400) A$, B: Microphotography after 3 days, $\mathrm{C}, \mathrm{D}$ : Microphotography after 7 days.

Seung-Woo Shin, et al: The Role of Collagen Membrane as a Scaffold of Etchant for Regional Acceleratory Phenomenon. J Kor Dent Sci 2011

consists of minerals.

$\mathrm{Kim}^{13)}$ reported that while acid demineralization didn't bring about the effect as much as the mechanical removal in corticotomy, though resorption of cortical bone, formation of new bone and osteoclasts activity in the demineralized region, which are also known as RAP, were observed. We assumed that the longer the acid was applied, the greater the demineralization effect was and decided to cover the flap without washing the acid to lengthen acid application time. To apply phosphoric acid without dispersion to adjacent tissues on a more efficient basis, we used absorbable collagen membranes. In general, there are two kinds of barrier membranes, absorbable and non-absorbable, and the latter's weak points include the need for secondary surgery to remove it and the difficulty of handling in clinic. To overcome these disadvantages, various absorbable membranes have been developed. Initially, absorbable barrier membranes were made of polylactic acid or polyglycolic acid; recently, there have been developments and studies on absorbable membranes made of type- 1 or type- 3 collagens from pigs or cattle. Collagen is characterized by chemotaxis for fibroblasts, hemostatic effect and low antigenicity; and acts as a scaffold for migrating cells ${ }^{18)}$. Despite these benefits, absorbable membranes composed of collagen also have limitations. When used in clinical field, they lack sufficient physical strength to make a space; when exposed to the oral cavity early, they are absorbed too quickly and allow infections, or lose the function as a barrier membrane. 
In addition, a barrier membrane should meet the conditions such as biocompatibility, histocompatibility, cell occlusivity, space-making function, ability to deliver nutrients, easiness of manipulation ${ }^{19)}$. While non-absorbable membranes mostly satisfy above conditions and have proven effectiveness $^{20)}$, absorbable membranes are not solid compared to non-absorbable membranes, making it difficult to maintain space for a required time ${ }^{21)}$. Collagen membrane used as a scaffold in the experiment absorbs blood and wound exudates; and shows good adsorption in wet conditions; it's adhesion is easy to handle; shows no deformation of product structure even when soaked in liquid, maintaining its characteristics, making it appropriate to be used as a scaffold; however, it is assumed that the cohesion of collagen results in reduced acid demineralization when collagen membrane is applied with liquid acid as the membrane absorbs and holds the acid, not delivering the acid directly to the tissue.

Jean et al. ${ }^{22)}$ observed histological changes when no orthodontic force was applied after corticotomy, and reported that when corticotomy is performed, bone metabolism and osteoclasts activity increase, causing reduced trabecula and increased porosity. In this experiment, histological observation of demineralized bone showed results similar to the histological observation in corticotomy. The reduction of bone density is due to transient osteoporosis phenomenon, which does not affect the whole body but only locally on the surgery site and adjacent area where RAP is shown. During the period showing RAP, osteoclasts are increasingly activated along periodontal ligament and teeth mobility grows, a cause of accelerated tooth movement.

To observe the osteoclasts activity, TRAP staining was carried out and the number of TRAP positive osteoclasts was observed. It is shown that the degree of osteoclasts activity increases in the initial period ${ }^{23)}$. Observations with a light microscope revealed a slight amount of osteoclasts on the $3^{\text {rd }}$ day of observation; but as time went by, almost no osteoclast was found in both the experimental and control groups. While Yaffe et al. ${ }^{24)}$ reported that the metabolism of alveolar bone increased only by lifting gingival periosteal flap, Wilcko et al. ${ }^{8)}$ commented that bleeding is more important than bone incision in the region where corticotomy is to be performed. Simple surgical procedures such as incision of periosteum or bone drilling, or bone demineralization using acid were also believed to be able to replace corticotomy. Likewise, this experiment was to obtain the effects similar to those of corticotomy with minimum stimulation as seen above, instead of using an intense surgery such as corticotomy.

\section{Conclusion}

Acid demineralization was accomplished on the mandibular cortical bone by applying an absorbable collagen membrane as a scaffold with $37 \%$ phosphoric acid; experimental animals were sacrificed in 3 days, 7 days and 2 weeks; and the histological results were compared with the control group that had applied only phosphoric acid. The results are as following:

1. The longer phosphoric acid was applied, the greater the amount of cortical bone demineralization at the site of phosphoric acid application was; and

2. When using absorbable collagen membrane, demineralization amount was rather reduced.

It can be seen that the greater the exposure to phosphoric acid, the larger the demineralization amount; and that even though the experiment used absorbable collagen membrane as a scaffold to increase the effect of bone demineralization and prevent dispersion to adjacent tissues, the bone demineralization degree was decreased, showing the role and function of absorbable collagen membrane as a scaffold was weak.

\section{References}

1. Hashimoto F, Kobayashi Y, Mataki S, Kobayashi K, Kato Y, Sakai H. Administration of osteocalcin accelerates orthodontic tooth movement induced by a closed coil spring in rats. Eur J Orthod. 2001; 23: 535-45.

2. Soma S, Matsumoto S, Higuchi Y, Takano-Yamamoto T, Yamashita K, Kurisu K, Iwamoto M. Local and chronic application of PTH accelerates tooth movement in rats. J Dent Res. 2000; 79: 1717-24.

3. Tengku BS, Joseph BK, Harbrow D, Taverne AA, Symons AL. Effect of a static magnetic field on orthodontic tooth movement in the rat. Eur J Orthod. 2000; 22: 475-87.
4. Nishimura M, Chiba M, Ohashi T, Sato M, Shimizu Y, Igarashi K, Mitani H. Periodontal tissue activation by vibration: intermittent stimulation by resonance vibration accelerates experimental tooth movement in rats. Am J Orthod Dentofacial Orthop. 2008; 133: 572-83.

5. Yamaguchi M, Hayashi M, Fujita S, Yoshida T, Utsunomiya T, Yamamoto H, Kasai K. Low-energy laser irradiation facilitates the velocity of tooth movement and the expressions of matrix metalloproteinase-9, cathepsin K, and alpha(v) beta(3) integrin in rats. Eur J Orthod. 2010; 32: 131-9.

6. Seifi M, Shafeei HA, Daneshdoost S, Mir M. Effects of two types of 
low-level laser wave lengths ( 850 and $630 \mathrm{~nm}$ ) on the orthodontic tooth movements in rabbits. Lasers Med Sci. 2007; 22: 261-4.

7. Kole H. Surgical operations on the alveolar ridge to correct occlusal abnormalities. Oral Surg Oral Med Oral Pathol. 1959; 12: 515-29.

8. Wilcko WM, Wilcko T, Bouquot JE, Ferguson DJ. Rapid orthodontics with alveolar reshaping: two case reports of decrowding. Int $\mathrm{J}$ Periodontics Restorative Dent. 2001; 21: 9-19.

9. Frost HM. The biology of fracture healing. An overview for clinicians. Part II. Clin Orthop Relat Res. 1989; (248): 294-309.

10. Frost HM. The biology of fracture healing. An overview for clinicians. Part I. Clin Orthop Relat Res. 1989; (248): 283-93.

11. Wilcko MT, Wilcko WM, Pulver JJ, Bissada NF, Bouquot JE. Accelerated osteogenic orthodontics technique: a 1-stage surgically facilitated rapid orthodontic technique with alveolar augmentation. J Oral Maxillofac Surg. 2009; 67: 2149-59.

12. Murphy KG, Wilcko MT, Wilcko WM, Ferguson DJ. Periodontal accelerated osteogenic orthodontics: a description of the surgical technique. J Oral Maxillofac Surg. 2009; 67: 2160-6.

13. Kim TG. Bony response to demineralization by etchant in the rabbits. Graduate School of Clinical Dental Science at Catholic University of Korea, 2010.

14. Kim T, Handa A, Iida J, Yoshida S. RANKL expression in rat periodontal ligament subjected to a continuous orthodontic force. Arch Oral Biol. 2007; 52: 244-50.

15. Bogra P, Kaswan S. Etching with EDTA--an in vitro study. J Indian Soc Pedod Prev Dent. 2003; 21: 79-83.

16. Legler LR, Retief DH, Bradley EL. Effects of phosphoric acid concentration and etch duration on enamel depth of etch: an in vitro study. Am J Orthod Dentofacial Orthop. 1990; 98: 154-60.

17. Wang Y, Spencer P. Effect of acid etching time and technique on interfacial characteristics of the adhesive-dentin bond using differential staining. Eur J Oral Sci. 2004; 112: 293-9.

18. Yaffe A, Ehrlich J, Shoshan S. Restoration of periodontal attachment employing enriched collagen solution in the dog. J Periodontol. 1984; 55: 623-8.

19. Scantlebury TV. 1982-1992: a decade of technology development for guided tissue regeneration. J Periodontol. 1993; 64(11 Suppl): 1129-37.

20. Kim CK, Cho KS, Chai JK, Choi EJ, Moon IS, Choi SH. Factors Influencing the wound healing in the periodontal intrabony lesion in human (I: Effect of the e-PTFE barrier membrane). J Korean Acad Periodontol. 1993; 23: 359-73.

21. Sandberg E, Dahlin C, Linde A. Bone regeneration by the osteopromotion technique using bioabsorbable membranes: an experimental study in rats. J Oral Maxillofac Surg. 1993; 51: 1106-14.

22. Jean DS, Alpdogan K, John WT, Robreto SC. Modeling of trabecular bone and lamina dura following selective alveolar decortications in rats. J Periodontal. 2008; 79: 1679-88.

23. Iino S, Sakoda S, Ito G, Nishimori T, Ikeda T, Miyawaki S. Acceleration of orthodontic tooth movement by alveolar corticotomy in the dog. Am J Orthod Dentofacial Orthop. 2007; 131: 448.

24. Yaffe A, Fine N, Binderman I. Regional accelerated phenomenon in the mandible following mucoperiosteal flap surgery. J Periodontol. 1994; 65: 79-83. 